\title{
Beam Selection Gain from Butler Matrices
}

\section{Citation}

Bai, Dongwoon, Saeed S. Ghassemzadeh, Robert R. Miller, and Vahid Tarokh. Beam selection gain from Butler matrices. In 2008 IEEE 68th Vehicular Technology Conference, 1-5. IEEE.

\section{Published Version}

doi:10.1109/VETECF.2008.107

\section{Permanent link}

http://nrs.harvard.edu/urn-3:HUL.InstRepos:2350493

\section{Terms of Use}

This article was downloaded from Harvard University's DASH repository, and is made available under the terms and conditions applicable to Open Access Policy Articles, as set forth at http:// nrs.harvard.edu/urn-3:HUL.InstRepos:dash.current.terms-of-use\#OAP

\section{Share Your Story}

The Harvard community has made this article openly available.

Please share how this access benefits you. Submit a story.

\section{Accessibility}




\section{Beam Selection Gain from Butler Matrices}

\author{
Dongwoon Bai* \\ dbai@fas.harvard.edu
}

\author{
Saeed S. Ghassemzadeh ${ }^{\dagger}$ \\ saeedg@ research.att.com
}

\author{
Robert R. Miller ${ }^{\dagger}$ \\ rrm@ research.att.com
}

\author{
Vahid Tarokh* \\ vahid@seas.harvard.edu
}

*Harvard School of Engineering and Applied Sciences, Cambridge, MA 02138, USA

${ }^{\dagger}$ AT\&T Labs. - Research, Florham Park, NJ 07932, USA

\begin{abstract}
We consider a wireless transmission scenario, when a base station is endowed with a fixed beamforming network, where $M$ antennas are employed at the base station to point beams to predetermined azimuthal angles. In our setting, a Butler matrix is deployed at the RF stage to form $M$ beams, and then the best beam is selected for transmission. We derive the distribution of the beam selection gain for this scenario under a Rician channel assumption as a function of both the azimuthal location of the remote unit and the Rician $K$-factor. Using some key properties of the noncentral chi-square distribution, we prove that beam selection outperforms antenna selection.
\end{abstract}

\section{INTRODUCTION}

We consider a wireless transmission scenario when a base station is endowed with $M$ multiple linear equally spaced omnidirectional array antennas and each remote unit is equipped with an omnidirectional antenna. The base station employs a fixed beamforming network (FBN) to improve on the signal quality by selecting the best beam for transmission to each remote unit among all the available fixed beams. The choice of the best beam can be achieved using a small amount of feedback information from the remote or by using the reverse link transmission (assuming channel reciprocity).

In the reverse link, we note that maximal ratio combining (MRC) at the base station is the optimal solution for the multiple receive antenna system. However, antenna selection is less complex because the antenna selection receiver only uses the antenna with the highest signal-to-noise ratio (SNR) and requires only one $\mathrm{RF}$ chain. In contrast, the MRC receiver requires $M \mathrm{RF}$ chains. Beam selection techniques are also attractive because only one RF chain is needed, in order to implement a FBN. The Butler matrix is a simple FBN at the RF stage producing orthogonal beams [1].

Although beam selection has been known to have no advantage over antenna selection in ideal Rayleigh fading channels, it has been established (using analysis and simulations) that beam selection can outperform antenna selection in correlated Rayleigh fading channels with limited angle spread [2]. For the case of Rician fading channels, no analysis exists except for two very special cases of Rayleigh fading channels and deterministic channels while simulations and measurements have shown that beam selection using a Butler FBN outperforms antenna selection [3].

Motivated by this, we analyze the performance of beam selection using a Butler FBN for Rician fading channels with arbitrary $K$-factors. We derive the exact distribution of the beam selection gain as a function of the azimuthal location of the remote user. Using this result, we compare the beam selection gain with the antenna selection gain for any Rician fading channel and analytically prove that beam selection is superior to antenna selection in all Rician channels.

The remainder of this paper is organized as follows: In Section II, we present our system model when a Butler FBN is used in the base station. In Section III, we analyze the beam selection gain using a statistical approach. In Section IV, we compare the gain of beam selection with that of antenna selection, and prove that beam selection outperforms antenna selection under any Rician channel transmission model. Finally, we provide our conclusions in Section V.

\section{The System Model}

We consider a base station endowed with $M \geq 2$ antennas (as depicted in Fig. 1) and remote units each endowed with one antenna.

In the reverse link, the $M \times 1$ received signal vector is given by

$$
\boldsymbol{r}=\boldsymbol{h} s+\boldsymbol{w}
$$

where the $M \times 1$ complex vector $\boldsymbol{h}=\left[h_{1}, \ldots, h_{M}\right]^{T}$ represents the flat fading channel gains normalized such that $\mathbb{E}\left[\left|h_{i}\right|^{2}\right]=$ 1 for $i=1,2, \cdots, M$, and the scalar $s$ is the transmitted signal. The noise vector $\boldsymbol{w}$ is modeled as an $M \times 1$ zeromean independent and identically distributed (i.i.d.) circularly symmetric complex Gaussian vector, with covariance matrix $\mathbb{E}\left[\boldsymbol{w} \boldsymbol{w}^{\dagger}\right]=\boldsymbol{I}_{M}$, where $\boldsymbol{I}_{M}$ is $M \times M$ identity matrix. In our model, the average transmit power is constrained to be $\mathbb{E}\left[|s|^{2}\right]=\rho$. This received signal is then processed by the Butler FBN whose $M \times 1$ output vector is given by

$$
\boldsymbol{y}=\boldsymbol{B r}=\boldsymbol{B h} s+\boldsymbol{B w},
$$

where $\boldsymbol{B}=\left[\boldsymbol{b}_{1}, \ldots, \boldsymbol{b}_{M}\right]^{T}$ denotes the $M \times M$ Butler matrix given by

$$
b_{m k}=\frac{1}{\sqrt{M}} e^{j \frac{2 \pi}{M}\left(m-\frac{1}{2}\right) k} .
$$

Clearly, the Butler matrix is unitary $\left(\boldsymbol{B}^{-1}=\boldsymbol{B}^{\dagger}\right)$ and can be considered as a generalized discrete Fourier transform (DFT) with offset $-1 / 2$. Since $\boldsymbol{B}$ is unitary, $\tilde{\boldsymbol{w}} \triangleq \boldsymbol{B} \boldsymbol{w}$ has the same distribution as $\boldsymbol{w}$. The output signal from the $m$-th port of the Butler FBN is the $m$-th entry of $\boldsymbol{y}$ in (2) and is given by

$$
y_{m}=\boldsymbol{b}_{m}^{T} \boldsymbol{h} s+\tilde{w}_{m} \text {. }
$$

For this port of Butler matrix, the SNR equals to $\rho \cdot \Gamma_{m}$, where

$$
\Gamma_{m} \triangleq\left|\boldsymbol{b}_{m}^{T} \boldsymbol{h}\right|^{2}
$$




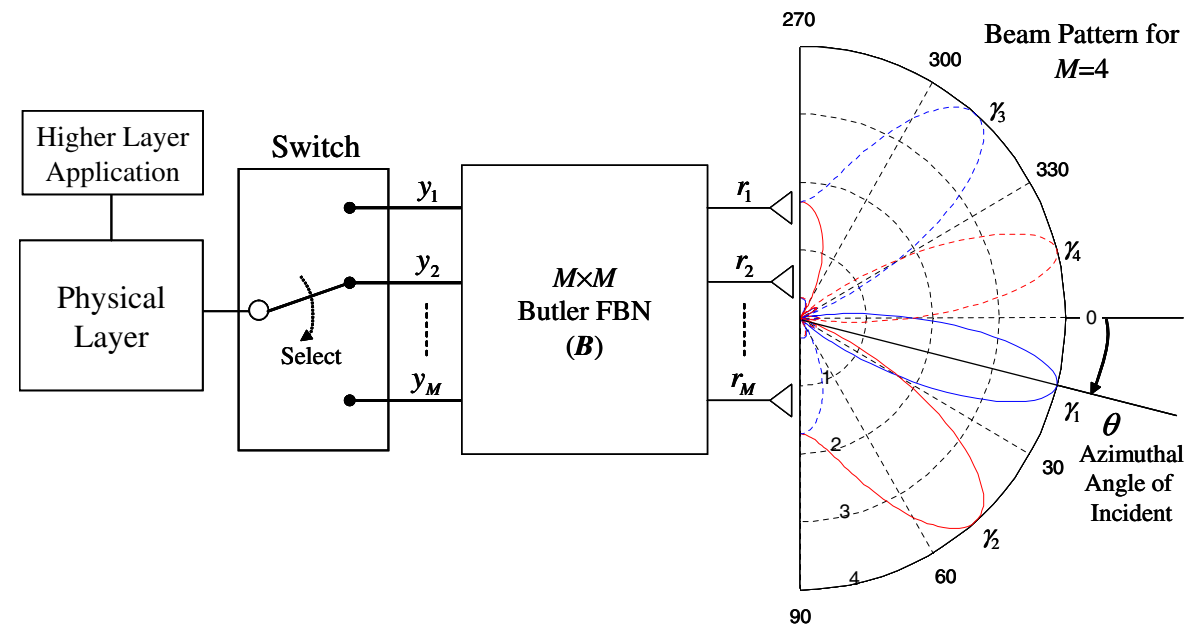

Fig. 1. Beam selection system using a Butler FBN with $M$ linear equally spaced array antennas and beam pattern for $M=4$ and $d=\lambda_{c} / 2$.

is the SNR gain of the $m$-th beam. The base station chooses the port with the highest SNR. The SNR is then given by $\rho \cdot \Gamma_{(M)}$, where the notation $z_{(m)}$ is used to denote the $m$-th smallest value from any set of finite samples $\left\{z_{1}, \ldots, z_{M}\right\}$, and thus $\Gamma_{(M)}=\max _{m \in\{1, \ldots, M\}} \Gamma_{m}$.

Next, we consider beam selection for the forward link. Assume that the base station sends the signal $s$ only through the $m$-th port of the Butler FBN. Using the same Butler FBN to feed the array, which is reciprocal, the signal vector fed to array is given by $\boldsymbol{b}_{m} s$. The received signal at the mobile user becomes

$$
y_{m}=\boldsymbol{h}^{T} \boldsymbol{b}_{m} s+w,
$$

which is mathematically identical with (4), and thus the SNR for this port is given by $\rho \cdot \Gamma_{m}=\rho\left|\boldsymbol{b}_{m}^{T} \boldsymbol{h}\right|^{2}$. To select the best beam, the remote user only needs to feedback the index of the best beam to the base station (even when the channel is not reciprocal) and this is the only difference between reverse and forward link beam selection. From this point on, we will not distinguish reverse and forward link beam selection in this paper as they are analytically identical.

We define the beam selection gain as the ratio of the SNR of beam selection with a FBN to the average SNR of random antenna switching without a FBN, which is given by $\Gamma_{(M)}$.

\section{Beam Selection Gains in Fading Channels}

It has been shown that beam selection outperforms antenna selection in ideal line-of-sight (LOS) channels, while beam selection performs as good as antenna selection in ideal nonline-of-sight (NLOS) channels [3]. We are interested in investigating the performance of beam selection under Rician channel models. This is the most frequently used realistic channel model in wireless communications. Under the Rician channel model, the normalized channel vector $\boldsymbol{h}$ can be modeled as multipath signals

$$
\boldsymbol{h}=\sqrt{\frac{K}{K+1}} \boldsymbol{h}_{L}+\sqrt{\frac{1}{K+1}} \boldsymbol{h}_{N} .
$$

The entries of complex vector $\boldsymbol{h}_{L}$ (which represents the normalized LOS component) are modeled to have unit power and fixed phase. The entries of the complex vector $\boldsymbol{h}_{N}$ (which represents the normalized NLOS component) are modeled by i.i.d. independent zero-mean circularly symmetric complex Gaussian random variables with unit variance. The parameter $K$ is referred to as the Rician $K$-factor, which represents the ratio of the LOS signal power to the NLOS signal power. The special cases of $K=\infty$ and $K=0$ represent ideal LOS (deterministic) and ideal NLOS (Rayleigh fading) channels, respectively.

\section{A. Deterministic Components}

Consider the LOS component $\boldsymbol{h}_{L}$. Let $\theta$ denote the azimuthal angle of incident between a LOS signal and the line perpendicular to the linear equally spaced array antennas assuming two-dimensional geometry (horizontal plane) as shown in Fig. 1. Furthermore, assume that the distance between the base station and the mobile user is much larger than array antenna separation. Then for both reverse and forward link beam selection, $\boldsymbol{h}_{L}$ is given by

$$
\begin{array}{r}
\boldsymbol{h}_{L}=\exp (j \psi)\left[1, \exp \left(-j 2 \pi \frac{d}{\lambda_{c}} \sin \theta\right), \ldots,\right. \\
\left.\exp \left(-j 2 \pi(M-1) \frac{d}{\lambda_{c}} \sin \theta\right)\right],
\end{array}
$$

where $\psi$ is an arbitrary phase shift of the signal from/to the first array antenna, $d$ is the distance between adjacent array antennas, and $\lambda_{c}$ is the carrier wavelength. 
Let the SNR gain of the $m$-th beam in ideal LOS channels $(K=\infty)$ be denoted by

$$
\begin{aligned}
\gamma_{m} & \triangleq\left|\boldsymbol{b}_{m}^{T} \boldsymbol{h}_{L}\right|^{2} \\
& =\left|\frac{1}{\sqrt{M}} \sum_{m=0}^{M-1} \exp \left(j m \phi_{m}\right)\right|^{2} \\
& = \begin{cases}M, & \text { if } \phi_{m}=2 \pi n, \quad n \in \mathbb{Z}, \\
\frac{1}{M} \frac{\sin ^{2}\left(M \phi_{m} / 2\right)}{\sin ^{2}\left(\phi_{m} / 2\right)}, & \text { otherwise, }\end{cases}
\end{aligned}
$$

where

$$
\phi_{m} \triangleq 2 \pi\left[\frac{1}{M}\left(m-\frac{1}{2}\right)-\frac{d}{\lambda_{c}} \sin \theta\right] .
$$

Since $\boldsymbol{h}_{L}$ is a function of $\theta, \gamma_{m}$ is also a function of $\theta$ and let us call a set of $M$ functions $\left\{\gamma_{m} \mid m=1, \ldots, M\right\}$ a beam pattern, which has the following properties:

$$
\begin{gathered}
\sum_{m=1}^{M} \gamma_{m}=M, \quad 0 \leq \gamma_{m} \leq M ; \\
\gamma_{m}=M \quad \text { if and only if } \quad \phi_{m}=2 \pi \frac{n}{M}, \frac{n}{M} \in \mathbb{Z} ; \\
\gamma_{m}=0 \quad \text { if and only if } \quad \phi_{m}=2 \pi \frac{n}{M}, \frac{n}{M} \notin \mathbb{Z} ;
\end{gathered}
$$

where (11) is from Parseval's theorem and the azimuthal angle satisfying (12) is the beam direction. Let us define a lobe of a beam as a main lobe if the beam direction is inside that lobe (although it can be called a grating lobe, we will not differentiate main and grating lobes). It can be shown that the condition for all $M$ beams to have at least one main lobe is given by

$$
\frac{M-1}{2 M}<\frac{d}{\lambda_{c}},
$$

which is usually assumed because there is no point in deploying $M$ antennas if some beams do not even have any main lobes. Furthermore, we can see that all $M$ beams have at most one main lobe in the half horizontal plane except its mirror in the other half if

$$
\frac{d}{\lambda_{c}}<\frac{M+1}{2 M}
$$

which may not be assumed if we want to exploit the diversity when the angular spread is not large enough. While the most common choice of the antenna separation $d$ is $\lambda_{c} / 2$ for each beam to have one main lobe, we only assume the condition (14) in this paper. Thus, each beam has at least one main lobe but it can also have multiple main lobes if the condition (15) is not met. Under (14), we can observe that the beam pattern is a periodic function of $\sin \theta$ with the period $\frac{\lambda_{c} / d}{M}$ and an even function of $\theta$. Since this paper neither evaluates the system based on some characteristic of $\theta$ nor analyzes the average performance based on the statistics of $\theta$, it is enough to examine the beam pattern from $\theta=0$ to the first beam direction given by

$$
\theta=\nu \triangleq \arcsin \left(\frac{1}{2 M} \frac{\lambda_{c}}{d}\right)
$$

\section{B. Probabilistic Analysis}

Now, let us consider the statistical channel model including NLOS components. Applying the Rician channel model in (7) to the SNR gain in (5) yields

$$
\Gamma_{m}=\left|\sqrt{\frac{K}{K+1}} \boldsymbol{b}_{m}^{T} \boldsymbol{h}_{L}+\sqrt{\frac{1}{K+1}} \tilde{h}_{N, m}\right|^{2}
$$

where $\tilde{\boldsymbol{h}}_{N}=\left[\tilde{h}_{N, 1}, \ldots, \tilde{h}_{N, M}\right]^{T} \triangleq \boldsymbol{B} \boldsymbol{h}_{N}$ is also a zero-mean i.i.d. circularly symmetric complex Gaussian random vector with unit variance for each entry since $B$ is a unitary matrix. Therefore, (17) becomes

$$
\begin{gathered}
\Gamma_{m}=\left(\sqrt{\frac{K}{K+1}} \Re\left\{\boldsymbol{b}_{m}^{T} \boldsymbol{h}_{L}\right\}+\sqrt{\frac{1}{K+1}} \Re\left\{\tilde{h}_{N, m}\right\}\right)^{2} \\
+\left(\sqrt{\frac{K}{K+1}} \Im\left\{\boldsymbol{b}_{m}^{T} \boldsymbol{h}_{L}\right\}+\sqrt{\frac{1}{K+1}} \Im\left\{\tilde{h}_{N, m}\right\}\right)^{2} \cdot
\end{gathered}
$$

Given that $\Re\left\{\tilde{h}_{N, m}\right\}, \Im\left\{\tilde{h}_{N, m}\right\}$ are independent and follow the zero mean Gaussian distribution with variance $1 / 2,2(K+$ 1) $\Gamma_{m}$ follows the noncentral chi-square distribution with two degrees of freedom and the noncentrality parameter

$$
\begin{aligned}
\delta_{m} & =\frac{\left(\sqrt{\frac{K}{K+1}} \Re\left\{\boldsymbol{b}_{m}^{T} \boldsymbol{h}_{L}\right\}\right)^{2}}{\frac{1}{2} \frac{1}{1+K}}+\frac{\left(\sqrt{\frac{K}{K+1}} \Im\left\{\boldsymbol{b}_{m}^{T} \boldsymbol{h}_{L}\right\}\right)^{2}}{\frac{1}{2} \frac{1}{1+K}} \\
& =2 K \gamma_{m},
\end{aligned}
$$

and thus the cumulative distribution function (cdf) of $\Gamma_{m}$ is given by

$$
\begin{aligned}
& F_{m}(x) \triangleq \operatorname{Pr}\left\{\Gamma_{m} \leq x\right\} \\
&=\left.F_{\chi^{\prime 2}}(2(K+1) x \mid n, \delta)\right|_{n=2, \delta=2 K \gamma_{m}} \\
&=\left.\mathbb{E}\left[F_{\chi^{2}}\left(2(K+1) x \mid n+2 P_{\delta / 2}\right)\right]\right|_{n=2, \delta=2 K \gamma_{m}}
\end{aligned}
$$

where $F_{\chi^{\prime 2}}(x \mid n, \delta)$ is the noncentral chi-square cdf with $n$ degrees of freedom and the noncentrality parameter $\delta, P_{\delta / 2}$ is a Poisson random variable with mean $\delta / 2$, and $F_{\chi^{2}}(x \mid q)$ is the chi-square cdf with $q$ degrees of freedom, given by

$$
\begin{aligned}
F_{\chi^{2}}(x \mid q) & =1-e^{-x / 2} \sum_{k=0}^{q / 2-1} \frac{(x / 2)^{k}}{k !} \\
& =e^{-x / 2} \sum_{k=q / 2}^{\infty} \frac{(x / 2)^{k}}{k !}
\end{aligned}
$$

if $q$ is an even number as in (20) where $q=n+\left.2 P_{\delta / 2}\right|_{n=2}$. Note that the cdf of $\Gamma_{m}$ is completely parameterized by $K$ and $\gamma_{m}$, and thus given $K$, evaluating $\gamma_{m}$ is enough to know the distribution of the SNR gain $\Gamma_{m}$. Therefore, we can focus on a set of $M$ random variables $\left\{\Gamma_{m} \mid m=1, \ldots, M\right\}$ only from $\theta=0$ to the first beam direction $\theta=\nu$ because the beam pattern $\left\{\gamma_{m}\right\}$ is a periodic function of $\sin \theta$ as described in the previous subsection. Since $\tilde{\boldsymbol{h}}_{N}$ is i.i.d., $\Gamma_{1}, \ldots, \Gamma_{M}$ are independent although they are not identical. Now, let us return 
to our primary interest, the beam selection gain $\Gamma_{(M)}$, whose distribution can be finally derived as

$$
\begin{aligned}
& F_{(M)}(x) \triangleq \operatorname{Pr}\left\{\Gamma_{(M)} \leq x\right\} \\
& \quad=\operatorname{Pr}\left\{\Gamma_{1} \leq x, \ldots, \Gamma_{M} \leq x\right\}=\prod_{m=1}^{M} F_{m}(x),
\end{aligned}
$$

and thus for $x>0$,

$$
\log F_{(M)}(x)=\sum_{m=1}^{M} \log F_{m}(x) .
$$

We have the following useful key theorem on the noncentral chi-square distribution, whose proof will be given in the full paper.

Theorem 1: The logarithm of the noncentral chi-square cdf with 2 degrees of freedom

$$
\log F_{\chi^{\prime 2}}(x \mid 2,2 \mu)
$$

is a strictly decreasing and strictly concave function of $\mu \geq 0$ for any given $x>0$ assuming that the base of logarithm is greater than one, where $\mu$ is half of the noncentrality parameter.

Now, we are ready to show the following theorem, where stochastic order relations are introduced in [4, Ch. 9].

Theorem 2: For any given $x>0, F_{(M)}(x)$, the cdf of the beam selection gain $\Gamma_{(M)}$, is a strictly decreasing function of $\theta$ from zero to the first beam direction $\nu=\arcsin \left(\frac{1}{2 M} \frac{\lambda_{c}}{d}\right)$. Therefore, in this interval, $\Gamma_{(M)}$ is stochastically increasing, stochastically smallest at $\theta=0$, and stochastically largest at $\theta=\nu$.

Proof: This proof will also be given in the full paper.

Fig. 2 confirms Theorem 2 showing that the distributions of the beam selection gain $\Gamma_{(M)}$ for various $\theta$ from zero to the first beam direction $\nu=7.18^{\circ}$. It can be clearly observed that $\Gamma_{(M)}$ is stochastically increasing as $\theta$ increases from zero to $\nu$ for $K=0,20 \mathrm{~dB}$.

The corollary below follows naturally from Theorem 2 .

Corollary 3: For $\theta \in[-\pi / 2, \pi / 2]$ and any integer $|m| \leq$ $\frac{M}{\lambda_{c} / d}, \Gamma_{(M)}$ is stochastically increasing as $\theta$ increases if

$$
\begin{aligned}
\theta \in & {\left[\arcsin \left(\frac{m}{M} \frac{\lambda_{c}}{d}\right),\right.} \\
& \left.\arcsin \left(\min \left\{\frac{m+1 / 2}{M} \frac{\lambda_{c}}{d}, 1\right\}\right)\right],
\end{aligned}
$$

and stochastically decreasing as $\theta$ increases if

$$
\begin{gathered}
\theta \in\left[\arcsin \left(\max \left\{\frac{m-1 / 2}{M} \frac{\lambda_{c}}{d},-1\right\}\right),\right. \\
\left.\arcsin \left(\frac{m}{M} \frac{\lambda_{c}}{d}\right)\right] .
\end{gathered}
$$

It is exactly opposite for the other half of the horizontal plane, $\theta \in[\pi / 2,3 \pi / 2]$. Therefore, $\Gamma_{(M)}$ with $\theta=0$ and $\theta=\nu$ are achievable stochastic lower and upper bounds, respectively for $\Gamma_{(M)}$ with an arbitrary $\theta$.

Corollary 3 tells us that the expected performance measures over $\Gamma_{(M)}$ with $\theta=0$ and $\theta=\nu$ can serve as lower and upper
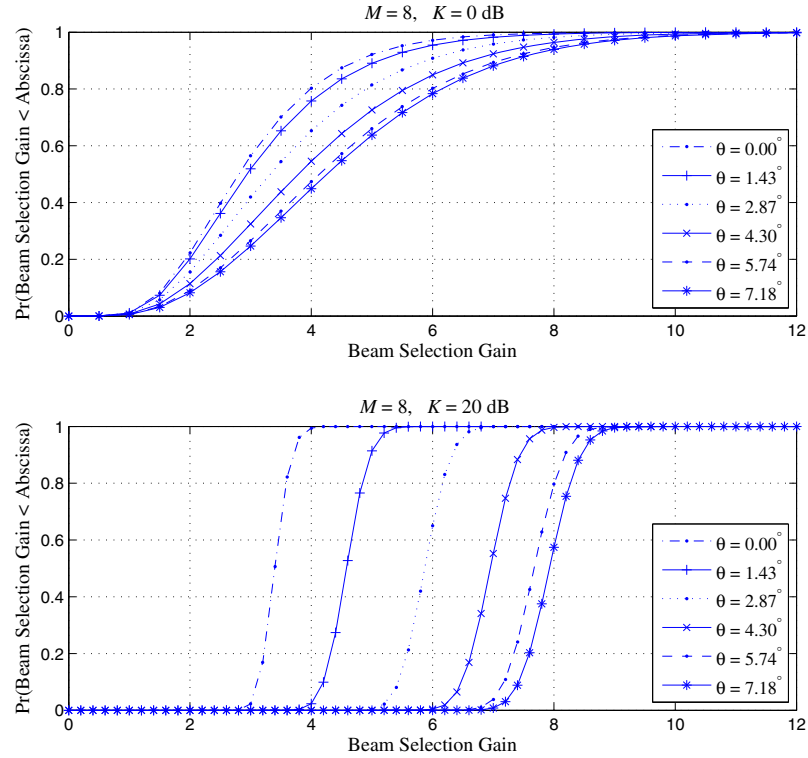

Fig. 2. Distributions of the beam selection gain $\Gamma_{(M)}$ for $M=$ $8, K=0,20 \mathrm{~dB}$, and various $\theta$ from zero to the first beam direction $\nu=7.18^{\circ}$, where $d=\lambda_{c} / 2$ is assumed.

bounds, respectively, for the averages of any performance measures which are increasing functions of SNR, e.g., the channel capacity. They can also serve as upper and lower bounds, respectively, for the averages of any performance measures which are decreasing functions of SNR, e.g., the bit error rate (BER), applying the result in [4, pp. 405-406].

\section{Beam Selection Versus antenna Selection}

Since one of the primary purposes for this work is to show the benefit of beam selection over antenna selection, let us consider the antenna selection gain under the same scenario used for beam selection case except the fact that the Butler FBN will not be deployed for antenna selection. When $m$-th antenna is selected among $M$ antennas in the base station, the SNR is given by $\rho \cdot H_{m}$, where $H_{m} \triangleq\left|h_{m}\right|^{2}$. Note that $H_{1}, \ldots, H_{M}$ are i.i.d. while $\Gamma_{1}, \ldots, \Gamma_{M}$ are just independent. Assuming that the antenna with the highest SNR is always selected, the antenna selection gain is defined as the ratio of the SNR of antenna selection to the average SNR of random antenna switching, which can be expressed by $H_{(M)}$. For any $m$, the cdf of $H_{m}$ becomes

$$
G(x) \triangleq \operatorname{Pr}\left\{H_{m} \leq x\right\}=F_{\chi^{\prime 2}}(2(K+1) x \mid 2,2 K)
$$

since it can be shown that $2(K+1) H_{m}$ follows the noncentral chi-square distribution with two degrees of freedom and the noncentrality parameter $2 K$. Therefore, the cdf of $H_{(M)}$ is given by

$$
G_{(M)}(x) \triangleq \operatorname{Pr}\left\{H_{(M)} \leq x\right\}=G^{M}(x) .
$$

Thus, the following lemma holds. 

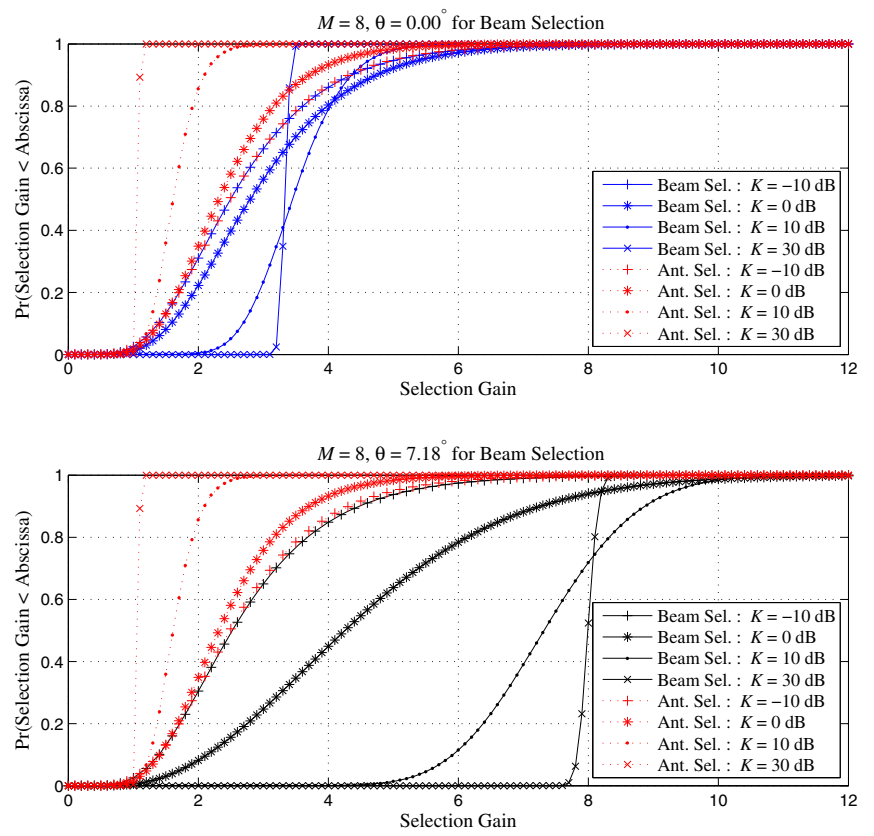

Fig. 3. Distributions of the beam selection gain $\Gamma_{(M)}$ and the antenna selection gain $H_{(M)}$ for $M=8, \theta=0^{\circ}, 7.18^{\circ}(=\nu)$, and $K=-10,0,10,30 \mathrm{~dB}$, where $d=\lambda_{c} / 2$ is assumed.

Lemma 1: For the same Rician $K$-factor, beam selection always outperforms antenna selection, i.e., the beam selection gain $\Gamma_{(M)}$ is stochastically larger than the antenna selection gain $H_{(M)}$.

Proof: Applying the concavity result in Theorem 1 and Jensen's inequality gives us

$$
\begin{aligned}
& \log G_{(M)}(x)=M \log F_{\chi^{\prime 2}}(2(K+1) x \mid 2,2 K) \\
& \geq \sum_{m=1}^{M} \log F_{\chi^{\prime 2}}\left(2(K+1) x \mid 2,2 K \gamma_{m}\right) \\
& =\log F_{(M)}(x),
\end{aligned}
$$

for any given $x>0$.

Fig. 3 shows the comparison of the beam selection gain $\Gamma_{(M)}$ and the antenna selection gain $H_{(M)}$ for various $K$ and $\theta$ of zero and the first beam direction $\nu=7.18^{\circ}$. Each group of the curves for the antenna selection gain is repeated for comparison in each subplot. We observe that for the same Rician $K$-factor, beam selection outperforms antenna selection, as proved in Lemma 1.

\section{CONClusion}

We considered a wireless transmission scenario, when a base station is endowed with a fixed beamforming network where $M$ antennas are employed at the base station to point beams to predetermined azimuthal angles. We derived the distribution of the beam selection gain for the Butler FBN and this distribution was given as a function of the azimuthal location of the user under Rician channel condition. We derived stochastic monotonicity results and stochastic bounds on the beam selection gain. We also proved that beam selection outperforms antenna selection. Graphical results were provided demonstrating the underlying gains.

\section{REFERENCES}

[1] J. Butler and R. Lowe, "Beam-Forming Matrix Simplifies Design of Electrically Scanned Antennas," Electronic Design, vol. 9, pp. 170-173, April 1961.

[2] Y.-S. Choi and S. M. Alamouti, "Approximate Comparative Analysis of Interference Suppression Performance between Antenna and Beam Selecion Techniques," IEEE Transactions on Wireless Communications, vol. 5, no. 9, pp. 2615-2623, Sep. 2006.

[3] A. Grau, J. Romeu, S. Blanch, L. Jofre, and F. D. Flaviis, "Optimization of Linear Multielement Antennas for Selection Combining by Means of a Butler Matrix in Different MIMO Environments," IEEE Transactions on Antennas and Propagation, vol. 54, no. 11, pp. 3251-3264, Nov. 2006.

[4] S. M. Ross, Stochastic Processes, 2nd ed., John Wiley \& Sons, 1996. 